\title{
A Novel Optimization of Cloud Instances WITH INVENTORY THEORY APPLIED ON REAL TIME IOT DATA OF STOCHASTIC NATURE
}

\author{
Mr. Sayan Guha \\ Data Architect, AI and Analytics Practice, Cognizant Technology Solutions, India
}

\begin{abstract}
A Horizontal scaling is a Cloud architectural strategy by which the number of nodes or computers increased to meet the demand of continuously increasing workload. The cost of compute instances increases with increased workload \& the research is aimed to bring an optimization of the reserved Cloud instances using principles of Inventory theory applied to IoT datasets with variable stochastic nature. With a structured solution architecture laid down for the business problem to understand the checkpoints of compute instances - the range of approximate reserved compute instances have been optimized \& pinpointed by analysing the probability distribution curves of the IoT datasets. The Inventory theory applied to the distribution curves of the data provides the optimized number of compute instances required taking the range prescribed from the solution architecture. The solution would help Cloud solution architects \& Project sponsors in planning the compute power required in AWS® Cloud platform in any business situation where ingestion \& processing data of stochastic nature is a business need.
\end{abstract}

\section{KEYWORDS}

AWS, Amazon Web Services, Inventory Theory, Reserved Instance, On-Demand Instance, Uniform Distribution, Gaussian Distribution, Poisson Distribution.

\section{INTRODUCTION}

The background of doing the research lies in the works of Sidney Brown et al [1] in his paper concerned with $(r, q)$ inventory model interpreted where demand accumulates continuously the demand rate at any instant is determined by an underlying stochastic process.

Also, Andrea Nodari [2] in his master's degree thesis has aimed to answer the few research questions, one of them being the modelling the cost optimization in Cloud Computing with Inventory theory. I propose to apply this cost optimization theory into real-time datasets \& for the same have chosen data from IoT devices which are stochastic \& varying in nature. My objective in the paper has been to understand the background of the solution architecture required to ingest, process \& store IoT datasets into AWS ${ }^{\circledR}$ platform \& then optimize the same by interpreting the distribution curves of IoT data captured on Cloud.

The technical challenge to the business problem lies in interpreting IoT data probability distribution curves on which Inventory theory has been applied \& interpreted of the distribution to optimize the compute instance (e.g., uniform distribution, Gaussian distribution curves being interpreted in my work with the data captured from IoT devices) 
I have considered Amazon Web Services as the chosen Cloud provider and to establish the concepts of Inventory theory applicability in optimizing the number of Amazon Elastic Compute Cloud (EC2) computing resources.

In this paper, my aim was to perform a validation exercise using a dataset emanated from an IoT device(s) and capture the many iterations of the data in a database to validate the stochastic nature of the source of data \& understanding the distribution of the data \& I believe this research will provide a foundation on which a dynamically time continuous stochastic data ecosystem can be assessed and provided with the optimized plan for instances.

The sections of the paper are as follows. In Section 2, I have provided background research work already established in this field. In Section 3, provides a brief background of different probability distributions, knowing which would help to understand my use case in the experiment section. In Section 4, my aim is to provide the high-level solution approach to the business problem. In Section 5, I have provided, the solution design using AWS® services which helps me approximately compute the expected range of Reserved Instances required for the business problem in Section 6.

In Section 7, the experiment scenario is discussed with the approach denoting decision points of data results. Section 8, I have discussed the experiments results applied the Inventory theory on data visualization of the dataset for experiment after concluding the response of the data in terms of probability distribution of the response obtained from data. Section 9 the optimization applied to the results obtained for the 2 different IoT sources used for the experiment. Section 10 talks about my inference from the experiment and in Section 11, I have discussed the areas of improvement of my work. The summarization of my work in Section 12.

\section{RELATED WORK}

With reference to the application of Inventory Theory applied in Cloud Economics by eminent research work in the field contributed by Andrea Nodari [2] on his thesis paper derived the relationship between Reserved Instances and On-Demand instances to optimize the usage in Cloud platform of Amazon Web Services. This paper has considered Amazon ${ }^{\circledR}$ Web Services as the preferred cloud platform for the work.

To set a background of this work from where the application of Inventory Theory can be applied to our experiment, I have added the basic difference of the Reserved \& On-Demand instances available with Amazon ${ }^{\circledR}$ in the cloud platform of Amazon ${ }^{\circledR}$ Web Services. 
International Journal on Cloud Computing: Services and Architecture (IJCCSA)

Vol. 11, No. 1/2/3/4/5/6, December 2021

Table 1: Difference between Reserved \& On Demand EC2 Instances Source: Derived from Website, https://aws.amazon.com/

\begin{tabular}{|c|c|c|c|}
\hline $\begin{array}{l}\text { Demand } \\
\text { perspective }\end{array}$ & $\begin{array}{l}\mathrm{EC} 2 \text { instance } \\
\text { type }\end{array}$ & Requirement & 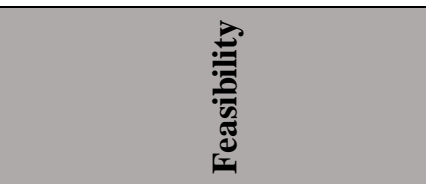 \\
\hline \multirow{6}{*}{$\begin{array}{l}\text { Demand is } \\
\text { predetermined } \\
\text { or agile }\end{array}$} & \multirow{3}{*}{ RESERVED } & UPFRONT & Predetermined \\
\hline & & HOURLY & Predetermined \\
\hline & & AD-HOC & $\mathbf{X}$ \\
\hline & \multirow{3}{*}{$\begin{array}{c}\text { ON- } \\
\text { DEMAND }\end{array}$} & UPFRONT & $\mathbf{X}$ \\
\hline & & HOURLY & Ad-hoc \\
\hline & & AD-HOC & Ad-hoc \\
\hline $\begin{array}{c}\text { Cost } \\
\text { Perspective }\end{array}$ & $\begin{array}{l}\mathrm{EC} 2 \text { instance } \\
\text { type }\end{array}$ & Requirement & 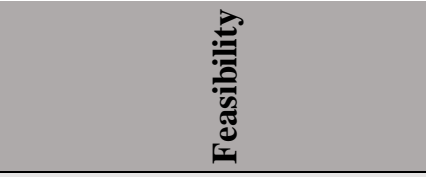 \\
\hline \multirow{6}{*}{$\begin{array}{l}\text { Cost \& } \\
\text { budget }\end{array}$} & \multirow{3}{*}{ RESERVED } & UPFRONT & Cheaper \\
\hline & & HOURLY & Cheaper when predetermined \\
\hline & & $\mathrm{AD}-\mathrm{HOC}$ & $\mathbf{X}$ \\
\hline & \multirow{3}{*}{$\begin{array}{c}\text { ON- } \\
\text { DEMAND }\end{array}$} & UPFRONT & $\mathbf{X}$ \\
\hline & & HOURLY & Cheaper when Ad-hoc \\
\hline & & AD-HOC & Cheaper when Ad-hoc \\
\hline
\end{tabular}

In the Inventory theory, for the sake of having an understanding between the reserved instances and on demand instances it has been considered.

$\mathrm{y}=$ Number of purchased reserved instances

$\mathrm{D}=$ Random variable representing the hourly demand of instances

$\mathrm{di}=$ ith observation of the demand $\mathrm{D}$

Cri (Count of Reserved Instances) < Cod (Count of On-demand Instances)

Total Cost $=\sum^{\mathrm{N}}(\mathrm{Cri \textrm {y }}+\mathrm{Cod} \max \{0, \mathrm{di}-\mathrm{y}\})$

$\mathrm{i}=1$

$=\sum C_{m}^{N} \ldots$ (when the company does not purchase any reserved instance)

$\mathrm{i}=1$

$\mathrm{N}$

$=\mathrm{N} \cdot \mathrm{y}$ (conversely when the purchase of reserve instance is more)

$\mathrm{i}=1$ 


\section{Explanation}

I assumed cost express in terms of random variable D. To minimize the total cost, the goal of the next step is to find the optimal value for $y$, the number of reserved instances to purchase. Hence the cost with demand $\mathrm{D}$ and $\mathrm{y}$

$\mathrm{C}(\mathrm{D}, \mathrm{y})=\mathrm{Cri} \mathrm{y}+\mathrm{Cod} \max \{0, \mathrm{D}-\mathrm{y}\}$

$\mathrm{C}(\mathrm{D}, \mathrm{y})$ is a random variahle it is possible to calculate the cost as

$$
\begin{aligned}
\mathbf{C}(\mathbf{y})=\mathbf{E}[\mathbf{C}(\mathbf{D}, \mathbf{y})] & =\sum_{\mathrm{d}=0}^{\infty}(\text { Cri } \mathrm{y}+\operatorname{Cod} \max \{0, \mathrm{~d}-\mathrm{y}\}) \operatorname{PD}(\mathrm{d}) . \\
& ={ }_{-}^{\infty} \quad(\mathrm{d}-\mathrm{y}) \operatorname{PD}(\mathrm{d}) \ldots \ldots \ldots \ldots \ldots \ldots \ldots \ldots \ldots \ldots
\end{aligned}
$$

I have approximated the dis ${ }^{\mathrm{d}=\mathrm{y}}$ ndom variable $\mathrm{D}$ with a continuous random variable such that $\Psi D(\xi)=$ Probability density function of D

$\Phi \mathrm{D}(\mathrm{a})=$ Cumulative distribution function $\mathrm{D}$ and the total cost is expressed as

$$
=\int_{0}^{a} \psi D(\xi) d \xi
$$

The total cost from expression...(i) now expressed as

$$
\begin{aligned}
& \mathrm{C}(\mathrm{y})=\mathrm{E}[\mathrm{C}(\mathrm{D}, \mathrm{y})]=\int_{0}^{\infty} C(\xi, y) \Psi D(\xi) d \xi \\
& \left.=\int_{0}^{\infty} \text { Cri y }+ \text { Cod } \max \{0, d-y\}\right) \boldsymbol{\Psi D}(\xi) d \xi \\
& =\operatorname{Cri~y~} \int_{\mathbf{0}}^{\infty} \operatorname{Cod}(\xi-\mathbf{y}) \boldsymbol{\Psi D}(\xi) d \xi
\end{aligned}
$$

To minimize the above equation quiet expectedly I have taken a derivative of expression...(iv) and set it to zero which has been further derived to

$$
\begin{aligned}
& \mathrm{dC}(\mathrm{y}) / \mathrm{dy}=\mathrm{Cri}-\operatorname{Cod}+\operatorname{Cod} \int_{0}^{y} \Psi D(\xi) d \xi=0 \\
& =\operatorname{Cri}-\operatorname{Cod}\left(1-\int_{0}^{y} \Psi D(\xi) d \xi\right)=0 \\
& =\mathrm{Cri}-\operatorname{Cod}[1-\Phi \mathrm{D}(\mathrm{y})]=\mathbf{0} \ldots . . .(\mathrm{v})[\text { as applied from above } \Phi \Phi(\mathrm{D}) \\
& \Phi D(y)=(\operatorname{Cod}-\text { Cri) } / \text { Cod ................................(iv) }
\end{aligned}
$$

In subsequent sections of this paper, I have applied the above derivation and relationship between cost of on demand instances and Cost of reserve instances \& used the same into IoT dataset used for the experiment 
International Journal on Cloud Computing: Services and Architecture (IJCCSA)

Vol. 11, No. 1/2/3/4/5/6, December 2021

\section{Probability Distributions: A BaCkground}

The theoretical background of 3 different probability distributions in the below table. Since the nature of the IoT data considered for the experiment henceforth is not pre-deterministic but stochastic in nature, therefore a background of understanding different probability distributions will help to interpret the dataset under consideration when the data is visualized in subsequent sections.

Table 2: Definition \& example(s) of different probability distributions e.g., Uniform, Gaussian \& Poisson distribution

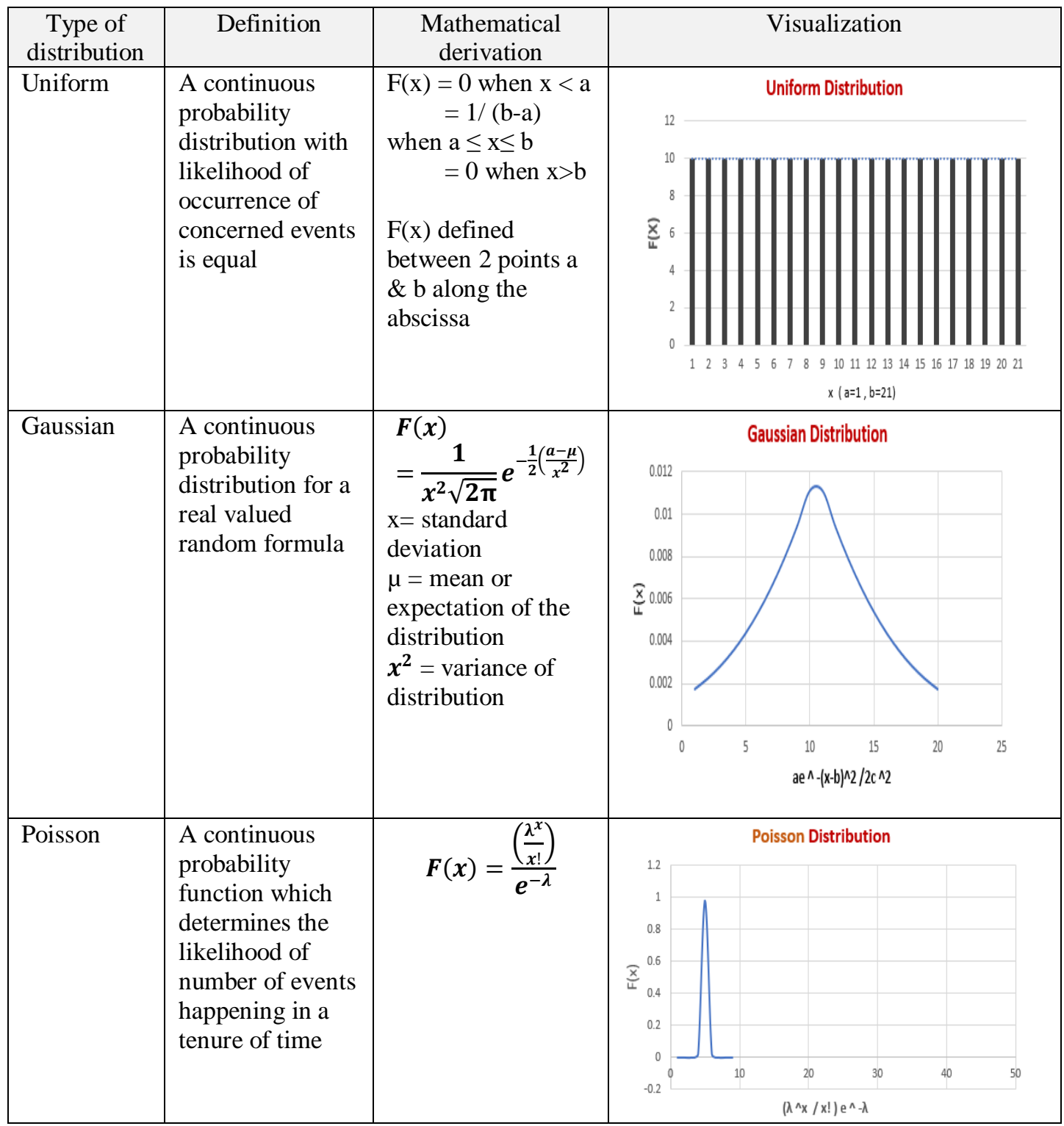




\section{SOLUTION APPROACH}

I propose below solution approach to the problem statement of optimization of Cloud instances considering an IoT data of stochastic distribution nature.

In the subsequent section the experiment would be discussed to arrive to the results for the dataset considered for experiment.

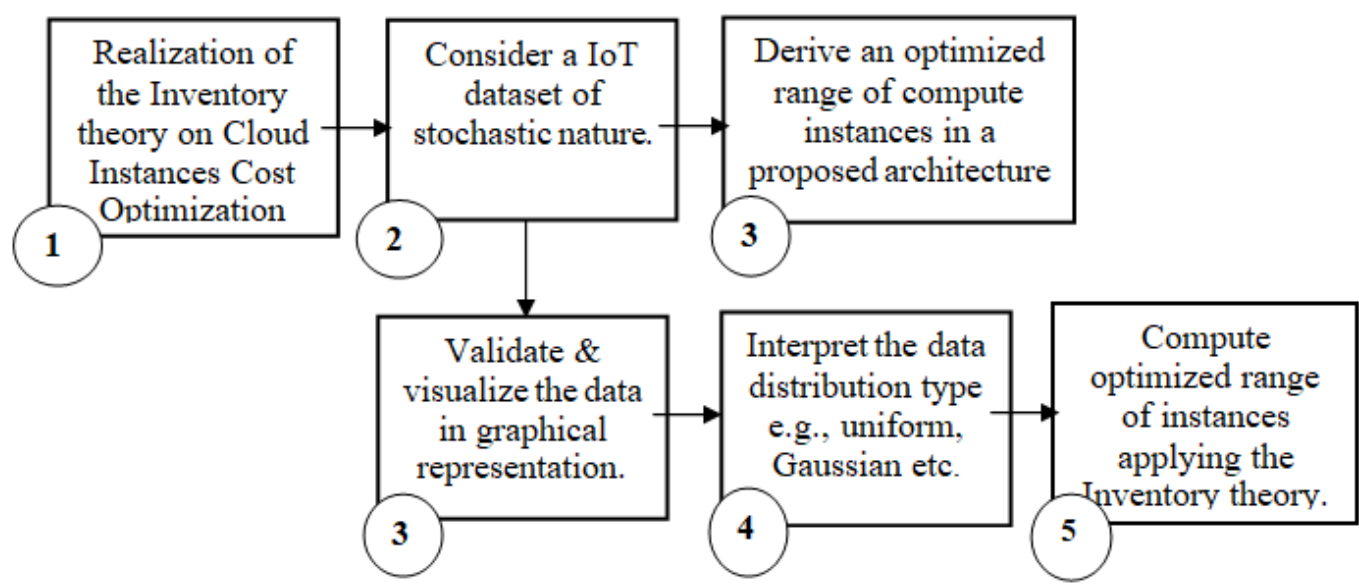

Figure 1. Solution approach flowchart with numbers denoting steps in the experiment (streams on step -3 are executed in parallel)

\section{Solution Design}

The dataset for the experiment to follow has been taken from open-source data repository available with

\subsection{Source of Data}

Dataset contains Beach Water Quality - Automated Sensors.

(https://data.world/cityofchicago/beach-water-quality-automated-sensors)

\subsection{Architecture Requirement}

- Capture the IoT data from the 3 IoT sensor starting from the edge location.

- Store the 'hot' data \& make it available for analysts /operational users.

- Feed the data through stream analytics system and make it available for any web application to access the data.

- Ensure high availability\& security using Virtual private Cloud environment.

\subsection{Solution Architecture Diagram}

I have visualized a solution architecture as below to capture the data from the above dataset $\&$ catering to requirement $\&$ in subsequent Table 3 have referred the points in the architecture where compute resources would be required. 


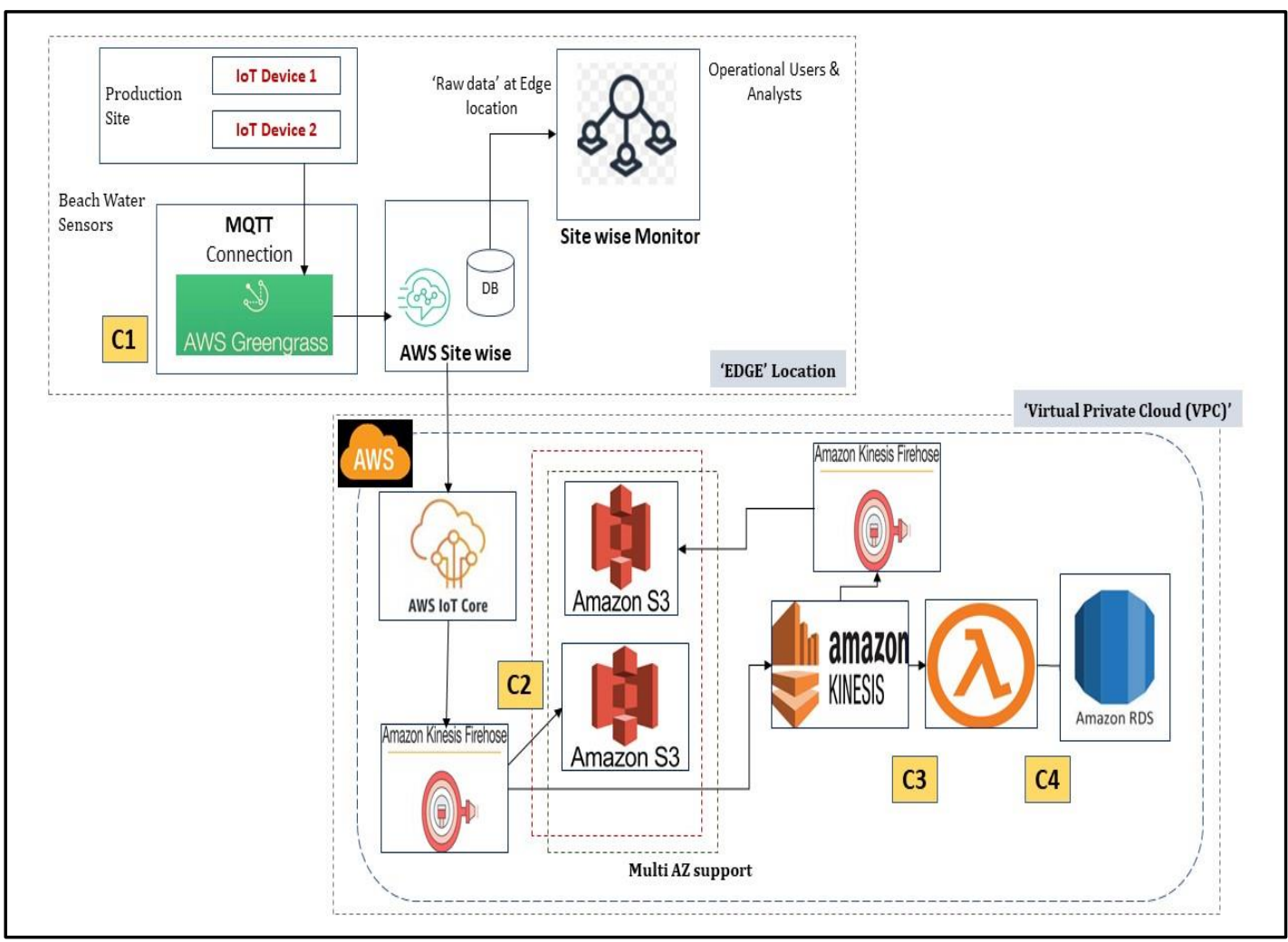

Figure 2: Preferred Solution architecture on Amazon ${ }^{\circledR}$ Web Service platform (architectural logos reference www.amazon.com)

\section{RANGE COMPUTATION OF INSTANCES}

The purpose of the solution architecture in this context was to determine the range of number of reserved instances that would be required to meet the business use case and which will be validated while applying the inventory theory in subsequent section of experiment based on the approximated number of reserved compute instances from Table-3 below.

\subsection{Considerations of Computation}

1. 2 separate Compute instances will be provisioned either by AWS managed service or by user of 2 separate IoT device to capture Beach Water Temperature \& Turbidity at every checkpoint of compute.

2. 2-3 Availability zone is considered for disaster recovery or high availability which would replicate the compute instance across different geographical zones \& provide a range of compute instances

3. The compute services used in the architecture are serverless, hence need not be provisioned or maintained separately, AWS underlying architecture would provision \& maintain the EC2 (Elastic Compute cloud instances- however, instances will be provisioned for computation \& that would be the basis for validating the experiment in subsequent sections 
International Journal on Cloud Computing: Services and Architecture (IJCCSA)

Vol. 11, No. 1/2/3/4/5/6, December 2021

Below results are obtained from looking into the solution from a bird's eye view of the data architecture.

Table3: Range Computation of Provisioned Compute Instances based on the preferred Solution Architecture

\begin{tabular}{|c|c|c|c|c|c|c|}
\hline \multicolumn{7}{|c|}{ Architectural Viewpoint of Range Computation of Instances } \\
\hline $\begin{array}{l}\text { Source } \\
\text { of } \\
\text { Data }\end{array}$ & $\begin{array}{c}\text { Compute } \\
\text { Checkpoint } \\
\text { No }\end{array}$ & $\begin{array}{c}\text { Compute } \\
\text { Checkpoint } \\
\text { Service } \\
\text { Name }\end{array}$ & Functionality & $\begin{array}{c}\text { No of } \\
\text { Instances } \\
\text { (A) }\end{array}$ & $\begin{array}{c}\text { No- } \\
\text { Multizone } \\
\text { Availability } \\
\text { (B) }\end{array}$ & $\begin{array}{c}\text { Approximate } \\
\text { number of } \\
\text { Compute } \\
\text { resources } \\
(\mathrm{A} * \mathrm{~B})\end{array}$ \\
\hline IoT & $\mathrm{C} 1$ & $\begin{array}{c}\text { Compute } \\
\text { instances } \\
\text { managed in } \\
\text { AWS } \\
\text { Greengrass } \\
\text { (Managed } \\
\text { service) }\end{array}$ & $\begin{array}{l}\text { Capture \& stream } \\
\text { IoT data for } \\
\text { device } 1 \& 2\end{array}$ & 2 & NA & 2 \\
\hline IoT & $\mathrm{C} 2$ & $\begin{array}{c}\text { Compute } \\
\text { instances } \\
\text { provisioned } \\
\text { by AWS } \\
\text { directing } \\
\text { stream data }\end{array}$ & $\begin{array}{l}\text { Direct Stream IoT } \\
\text { Data to } \mathrm{S} 3 \text { object } \\
\text { storage }\end{array}$ & 2 & $2-3$ & $4-6$ \\
\hline IoT & $\mathrm{C} 3$ & $\begin{array}{c}\text { AWS } \\
\text { Lambda } \\
\text { (Managed } \\
\text { service) }\end{array}$ & $\begin{array}{c}\text { Provisioned } \\
\text { Compute - } \\
\text { Function/ Code to } \\
\text { ingest stream data }\end{array}$ & 2 & $2-3$ & $4-6$ \\
\hline IoT & $\mathrm{C} 4$ & $\begin{array}{l}\text { Instances } \\
\text { provisioned } \\
\text { for RDS } \\
\text { service } \\
\end{array}$ & $\begin{array}{l}\text { Stores data for } \\
\text { further analysis \& } \\
\text { querying purpose }\end{array}$ & 2 & $2-3$ & $4-6$ \\
\hline \multicolumn{6}{|c|}{ Approximate Range of Compute Instances } & 14-20 \\
\hline
\end{tabular}

\section{Explanation of above Range approximation}

- Checkpoint C1, C2, C3 \& C4 corresponds to my solution architecture in Figure 4. Corresponding to the compute instances managed by AWS Greengrass, compute capacity used to direct the data to AWS S3, provisioned compute- Function/ Code to ingest stream data in AWS Lambda managed service \& processing the final data set to RDS respectively.

- 2 separate IoT device to capture Beach Water Temperature \& Turbidity at every checkpoint of compute \& hence compute instance (whether managed by AWS or manually provisioned) have been assumed to be 2 for all checkpoints \& hence with 4 compute checkpoints -I approximate the probable usage of 8 instances which need to be reserved upfront for one availability zone

- At the Edge Computing zone, AWS Greengrass doesn't need the data to be available across availability zones but for all other compute points data need to high available which I propose to be 2-3 multizone availability based on requirement of consumption.

- Therefore, I have done a range approximation of Compute instances required in the solution as per the Table 3 considering only multizone availability of 6 compute instances $(\mathrm{C} 2, \mathrm{C} 3, \& \mathrm{C} 4)$ with 2-3 multizone availability which makes it 12-18 compute 
instances+ 2 instances considered at edge with AWS Greengrass makes the range as 1420 compute instances to be reserved upfront from solution architect point of view.

When I moved to the subsequent section of the paper to interpret the IoT data from a probability distribution perspective, my goal was to optimize the range as already computed above \& specify the actual number of instances required in this process of interpretation of data using Inventory theory applied to response probability distribution of the datasets.

The value adds, I proposed will support the contextual solution architecture approximation of compute resources assume a range of compute instances at the very high level of solution based on assumptions to streamline \& pinpoint the number of instances by interpretation of actual data response applying Inventory theory applied to their probability distributions.

\section{Experiment Scenario: Probability Distribution of Data SOURCE}

Aiping Wang et al [3] in their publication of Survey on stochastic distribution systems found the the control task is to obtain control signals so that the output Probability Distribution functions of stochastic systems are made to follow their target Probability Distribution functions The research in this field already established by predecessors me to formulate the probability distribution function for the IoT data under consideration as the initial step.

I have observed with solution architecture defined for the ingestion of IoT data from 2 devices specific to the use case could provide a high-level estimation of compute resources required for the requirement under consideration. In this context, would like to clarify that the 2 IoT devices would capture 2 specific attributes of the data generated at the site namely 1) Water temperature $\& 2)$ Turbidity.

In this process of performing the experiment I have analysed the work of D. Altman et al [4] which took samples of various probability data distributions samples \& interpreted it through various methods of analysis make assumptions about normality, including correlation, regression, tests, and analysis of variance. It has also been concluded is not in fact necessary for the distribution of the observed data to be normal, but rather the sample values should be compatible having a normal distribution in defined range of values.

Peter Jez [5] in his technical paper compared multiple data samples and carried on a series of mathematical test to conclude, mathematically Normal Distribution would be range bounded at its boundaries but normal distribution in Gaussian density distribution will have a mean or expectation of the value exponentially raised and divisible by the variance of distribution.

Based on all the above studies in the field of probability distribution -the below approach has been devised to arrive to the interpretation of the results as per the flow chart. 


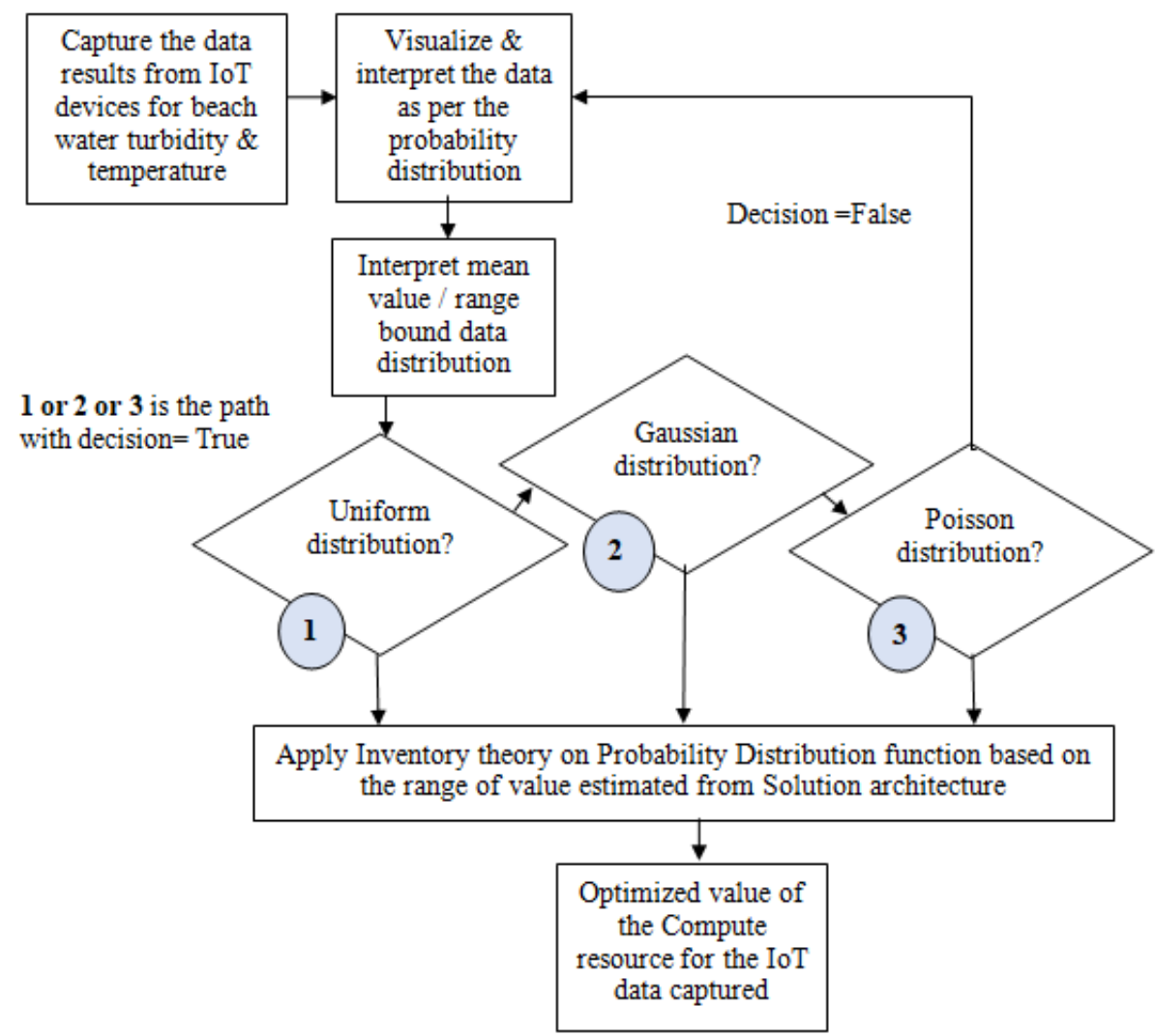

Figure 3: Experiment Approach flowchart with numbers denoting decision points of interpretation of data results in the experiment

\section{EXPERIMENT Results: Data Visualization}

The experiment results visualizing the data output captured for Water Temperature \& Turbidity of Beach water in Ohio shows

- IoT Sensor1 Output (Water Temperature): As observed, the temperature is rangebound 12 degrees Fahrenheit to 27 degrees Fahrenheit with a mean $(\boldsymbol{\mu})=\mathbf{4 2}$ around within the range of 19-20 degrees. Instances were captured real time for7500 timestamp instances from IoT sensor 1, ingested in the RDS database instances and presented as below Figure -4 .

- IoT Sensor1 Output (Turbidity): As observed, the turbidity readings were slowly taking peak as observed around the value of 42 Formazin Turbidity Unit (FTU) \& gradually declining with being normalized - the distribution fairly forms a bell-curve along the value of mean $(\boldsymbol{\mu})=\mathbf{4 2}$ with standard deviations $(-x) \sim 25$ FTU \& standard deviation (+x) $\sim 25$ FTU equally spaced from the mean. Trend lines have been drawn trend lines along both the observations. 


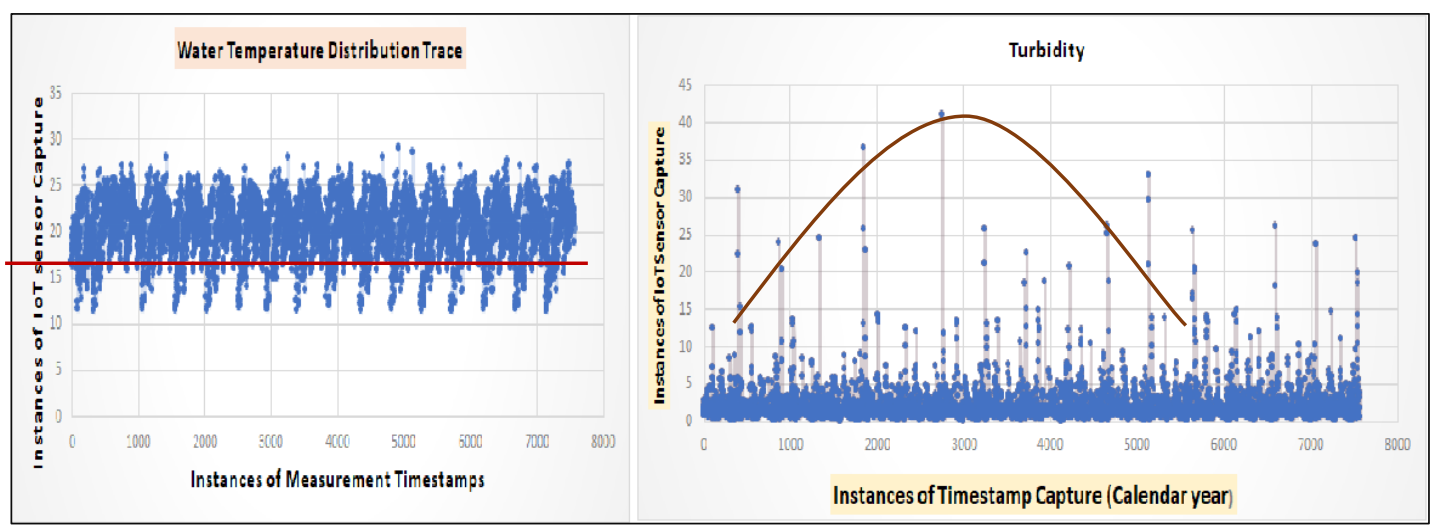

Figure 4: Data results in the experiment-Depiction of Water Temperature \& Turbidity captured across timestamp ranges (Left - Water temperature Vs Timestamp, Right- Turbidity Vs Timestamp)

\section{Inference}

- The probability distribution of IoT devicel is inferred as Normal Distribution

- The probability distribution of IoT device1 is inferred as Gaussian Distribution

I have taken an assumption to give equal weightage to the IoT data source considering the requirement of Cloud Compute resource while applying Inventory Theory for optimization of forecasted range of computer resources.

The end goal is optimizing the total number of compute resources possibly could be required in this process of capturing IoT real time data of stochastic nature with the help of Inventory theory discussed in Section-2 of this paper \& the baseline range would be considered as calculated from Section-6, Figure -3

\section{EXPERIMENT RESULT: OPTIMIZATION BY APPLYING INVENTORY THEORY}

The baseline range outlined would be considered as calculated from Section-3, Figure -3 considering approximately 2-3 availability zones \& the solutions has been architected using the solution architecture in Figure-2. The services invoked in the solution are in many cases managed services of Amazon (B) Web Services but each of the services on the background invokes instances for the best suitability of the IoT data processing under consideration.

The best of the solution architecture design could be done with an approximation with the solution architecture under consideration which have considered standard well architected framework of Amazon ${ }^{\circledR}$ Web Services particularly suited for the requirement Luis A. San-José et al [6] have used the Inventory theory to maximize profit in an inventory system of Time varying demand, I have applied the same concept of applying Inventory theory into time varying data to maximize resource utilization.

I have investigated the problem statement from the data perspective by which applied statistical analysis \& pure probability distribution by interpreting the nature of the data \& output produced from the IoT sensors as depicted and visualized in Figure-4. 


\subsection{Optimization of Instances for IoT Sensor 1}

Assuming equal weightage on distribution of compute instances baselined in Section-3 I have considered $50 \%$ of the instances (7-10 instances) to be consumed to process the IoT Sensor 1 data which measures Water Temperature Vs Timestamp.

The output has been inferred to be producing a Normal Probability Distribution

Hence, the probability distribution function $\&$ the cumulative distribution function in the interval of $[\mathrm{m}, \mathrm{n}]$ can be defined as

\section{Density function:}

$$
\begin{aligned}
& \Psi(\xi)=0 \text { if } \xi<\mathrm{m} \\
& \Psi(\xi)=1 /(\mathrm{n}-\mathrm{m}) \text { if } \mathrm{m} \leq \xi \leq \mathrm{n} \\
& \Psi(\xi)=0 \text { if } \xi>\mathrm{n}
\end{aligned}
$$

\section{Cumulative Distribution:}

$$
\begin{aligned}
& \Phi D(y)=0 \text { if } y<m \\
& \Phi D(y)=(y-m) /(n-m) \text { if } a \leq y \leq b \\
& \Phi D(y)=1 \text { if } y>n
\end{aligned}
$$

- Assuming the optimal number of reserved instances will be in the range of $\mathbf{7} \leq \mathbf{y} \leq \mathbf{1 0}$

- Assuming the consideration of partial upfront plan of AWS® with hourly rate for ondemand \& reserve instances of $\mathrm{m} 3$. medium instances in us region (us-east1-a or uswest 1-b) - Cost $/ \mathbf{h r}($ On demand $)=\$ 0.07 \&$ Cost $/ \mathbf{h r}($ Reserved $)=\$ 0.05$

$\Phi D(y)=(\operatorname{Cod}-$ Cri $) / \operatorname{Cod}$

$(\mathrm{y}-7) /(10-7)=(0.07-0.05) / 0.07=7.85$

$\mathrm{Y} \sim 8$ Instances.

\subsection{Optimization of Instances for IoT Sensor 2}

Assuming equal weightage on distribution of compute instances baselined in Section-3 I have considered $50 \%$ of the instances (7-10 instances) to be consumed to process the IoT Sensor 1 data which measures Turbidity Vs Timestamp.

The output has been inferred to be producing a Gaussian Probability Distribution

Following similar approach like above from Jamie Zappone[6] outlined that the probability distribution function \& the cumulative distribution function in the interval of $[\mathrm{m}, \mathrm{n}]$ can be defined as

\section{Density function:}

$$
\Psi(\xi)=\frac{1}{\sigma \sqrt{2 \Pi}} e^{-\left(\frac{\left.x-\mu^{\prime}\right)^{2}}{2 \sigma^{2}}\right.}
$$

\section{Cumulative Distribution:}


International Journal on Cloud Computing: Services and Architecture (IJCCSA)

Vol. 11, No. 1/2/3/4/5/6, December 2021

$$
\Phi \mathrm{D}(\mathrm{y})=\frac{1}{\sqrt[\sigma]{2 \Pi}} \int_{\infty}^{y} \boldsymbol{e} \frac{-(t-\mu)^{2}}{2 \sigma^{2}} d t
$$

- Assuming the optimal number of reserved instances will be in the range of $\mathbf{7} \leq \mathbf{y} \leq \mathbf{1 0}$

- Assuming the consideration of partial upfront plan of AWS ${ }^{\circledR}$ with hourly rate for ondemand \& reserve instances of $\mathrm{m} 3$. medium instances in US region (us-east1-a or uswest 1-b) - Cost $/ \mathbf{h r}($ On demand $)=\$ 0.07 \&$ Cost $/ \mathbf{h r}($ Reserved $)=\$ 0.05$

- The Mean $(\mu)$ is observed to be 42 , standard deviation $(\sigma)=60$ within the range of normalization of Min value $=2000$ and Max value $=4000$ in Figure 4 which calculates the Cumulative Distribution function $(\Phi D(y))$ for Gaussian distribution $\sim 0.285$ when the upper limit of integration $(\mathbf{y})=\mathbf{8}$ [ Calculated using Cumulative Dstribution Calculator for Gaussian distribution]

Referring to the Cost Optimization obtained from Inventory Theory model as below

$\Phi D(y)=(\operatorname{Cod}-\operatorname{Cri}) / \operatorname{Cod}$

$\boldsymbol{\Phi D}(\mathbf{y})=(0.07-0.05) / 0.07=0.285$ is true when

$\mathrm{y} \sim 8$ Instances.

The total number of reserved instances applying Inventory theory model for optimization $=(\mathrm{A})+(\mathrm{B})=16$.

\section{EXPERIMENT INFERENCE}

- In this work I have predicted an estimated number of total reserved compute capacity expected to process the IoT data from the site location emanating from 2 IoT sensors with a stochastic \& unpredictable nature of data propagation to be in the range of 14-20 instances depending on the number multi-availability zone under consideration.

- I have intended to perform the experiment on the Beach water data and interpreted the probability distribution of turbidity and water temperature of the beach water data. As observed the water temperature results could be interpreted as uniform probability distribution \& the turbidity as Gaussian distribution for thousands of instances of captured for every event. The Inventory theory model for optimization of reserved Cloud Compute resources was hence applied and found that number of instances optimized by Inventory model application is streamlined to $(\mathbf{8 + 8})=\mathbf{1 6}$ instances which falls correctly into the range of instances predicted $\&$ assumed during solution architectural approach.

\section{Limitations \& AREAS OF IMPROVEMENT}

While in the process of background study I analysed Kenneth J. Arrow et al [7] where optimal inventory policies have been discussed with constant \& variable rates of changes, I took my understanding to model the solution of variable data inflow in an idealized rather than an exact representation of the real problem. Hence my results do not guarantee the solution to be the best possible solution, rather an approach with real time data to achieve an optimized prediction of reserved compute instances $\&$ would lead to cost optimization with upfront plan of reserved instances. 


\begin{abstract}
Abayomi et al [8] discussed in their research paper on Dynamics of Inventory Cost Optimization the application of Inventory Theory model applied to both deterministic and stochastic demands $\&$ thereby arrived to find out the optimised stock procurement approach when applied in context of Supply chain. I have however, not performed any experiment to apply the Inventory Model to determine the number of reserved compute instances in any scenario where there is a constant deterministic in nature rather the concept of Inventory Model Cost optimization has been applied to stochastic data emanating from IoT \& captured in AWS ${ }^{\circledR}$ cloud architecture to predict a baseline of the number of reserved compute instances \& further optimized using the distribution output of the data sources.
\end{abstract}

Hence, I acknowledge the application of the same solution approach into data sources of constant deterministic data is a subject of further research.

\title{
12. Conclusion
}

I expect that my work to be a connecting between all the research work established to optimize the reserved capacity cost optimization in Cloud Computing. With real data obtained from IoT devices which have an uncertainty stochastic nature of propagation, I have intended to simulate a solution architecture to capture such data to predict a range of compute capacity required for flow, propagation \& storage \& for the same opted AWS ${ }^{\circledR}$ Cloud platform services. In my endeavour to further interpret the dataset into probability distribution curves I have applied Cost optimization from Inventory theory model to identify the optimized number of reserved instances required for the experiment performed which was observed to be falling in the range of instances predicted by solution approach.

I, trust this work will motivate upcoming avenues of future research where data is stochastic \& real time, data driven approach for cost estimation of compute power is necessary and budget for the same need to be predetermined with less dependence on the Cloud service provider to provide the provisioning plans.

\section{REFERENCES}

[1] Sidney Browne and Paul Zipkin (1991) "Inventory Models With Continuous, Stochastic Demands", https://www0.gsb.columbia.edu/mygsb/faculty/research/pubfiles/6353/browne_inventory_models.pdf

[2] Andrea Nodari (2015) "Cost Optimization - in Computing”https://aaltodoc.aalto.fi/bitstream/handle/123456789/17711/master_Nodari_Andrea_1970 .pdf?sequence $=1$

[3] Aiping Wang and Hong Wang (2021) "Survey on stochastic distribution systems: A full probability density function control theory with potential applications" https://www.ornl.gov/publication/surveystochastic-distribution-systems-full-probability-density-function-control-theory

[4] D. Altman \& J. Bland (1995) " Statistics notes: The normal distribution" https://pubmed.ncbi.nlm.nih.gov/7866172/

[5] Peter Jez (2012) "Uniform Distribution with respect to Gaussian measure" http://www.cosy.sbg.ac.at/research/tr/2012-02_Jez.pdf

[6] Luis A. San-José, Joaquín Sicilia, Manuel González-de-la-Rosa \& Jaime Febles-Acosta (2021) "Profit maximization in an inventory system with time-varying demand, partial backordering and discrete inventory cycle" https://link.springer.com/article/10.1007/s10479-021-04161-6

[7] Kenneth J. Arrow, Theodore Harris and Jacob Marschak (1958) "Optimal Inventory Policy" https://www.or.mist.i.u-tokyo.ac.jp/takeda/FreshmanCourse/ArrowHarrisMarschak.pdf

[8] T Abayomi, Onanuga, Adekunle Adeyemi (2014) "Dynamics of Inventory Cost Optimization-A Review of Theory and Evidence" https://www.iiste.org/Journals/index.php/RJFA/article/view/17593/0 
International Journal on Cloud Computing: Services and Architecture (IJCCSA)

Vol. 11, No. 1/2/3/4/5/6, December 2021

\section{AUTHOR}

Sayan Guha completed his Bachelors in Electronics \& Communication Engineering in 2006. He has been serving Information Technology industry supporting Data Modelling \& Architecture across business domains of Retail, Banking, and Insurance $\&$ Telecom. He is currently working with Cognizant technology Solutions and his area of interests are in the fields of Cloud Solution architecture, Big Data Integration, API based data integration \& Advanced analytics.

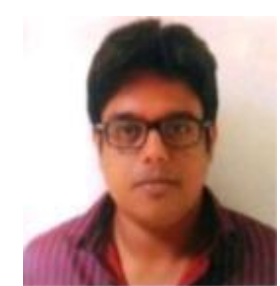

